\title{
REGULAR NEIGHBOURHOODS AND THE STABLE RANGE
}

\author{
BY \\ W. B. R. LICKORISH( $\left.{ }^{1}\right)$ AND L. C. SIEBENMANN
}

0. Introduction. We prove the following product theorem for regular neighbourhoods of a polyhedron in a P.L. (= piecewise linear) manifold and give a number of basic applications. The most difficult identifies a large (and in a sense maximal) class of noncompact P.L. manifolds that are determined up to P.L. homeomorphism by their ordinary homotopy type and stable tangent bundle.

Theorem 1 (Product Theorem). Let $e: X \times I \rightarrow M$ be a P.L. proper imbedding, where $X$ is a (possibly noncompact) polyhedron, $M$ is a P.L. n-manifold, $n \geqq \operatorname{dim}(X \times I)+3$, and $e^{-1}(\partial M)=X \times\{0,1\}$. If $P$ is a regular neighbourhood of $e(X \times I)$ in $M$ such that $P \cap \partial M$ is a union $P_{0} \cup P_{1}$ of regular neighbourhoods in $\partial M$ of $e(X \times 0)$ and $e(X \times 1)$ respectively, then there exists a P.L. homeomorphism $f: P_{0} \times I \rightarrow P$ such that

$$
e(x, t)=f(e(x, 0), t)
$$

and

$$
f\left(P_{0} \times i\right)=P_{i}, \quad i=0,1 .
$$

This is closely related to the proposition that any block bundle (see [13]) $\xi$ over $X \times I$ is isomorphic to $(\xi \mid X \times 0) \times I$. In fact, if $X$ is a P.L. manifold this is clearly equivalent, but for $X$ a complex they merely say similar things about quite different sorts of object.

Our proof consists of a pleasant reduction to a special case of a P.L. isotopy extension problem for a polyhedron in a manifold (of codimension $\geqq 3$ ). This special case is quickly disposed of with the cone unknotting theorem of [10]. (The general case, which is more difficult, is an unpublished result of J. F. P. Hudson.)

The remainder of the paper uses Theorem 1 as a tool to produce results that are more striking.

The seminar notes of E. C. Zeeman [19] form a general reference for P.L. topology. Where there is no indication to the contrary, the following conventions will hold in this paper. Simplicial complexes are locally finite and finite dimensional. A polyhedron is a possibly noncompact separable polyhedron of finite dimension. A manifold is a possibly noncompact separable P.L. manifold, possibly

Received by the editors February 14, 1968.

(1) Supported in part by NSF grant GP 7085 (144-7639). 
with boundary, and each component has the same finite dimension. All maps, homotopies, imbeddings, and immersions, etc., are both P.L. and proper. Proper means that the preimage of each compactum is compact.

$\S 2$ shows that if $Y \times I \subset M$ is a closed subpolyhedron of codimension $\geqq 3$ in a P.L. manifold, and meets $\partial M$ in $Y \times 0$, then there exists a P.L. imbedding $h: \partial M \times(I, 0) \rightarrow(M, \partial M)$ such that $h \mid(Y \times 0) \times I$ maps onto $Y \times I \subset M$ in the natural way and $h(m, 0)=m$ for $m \in \partial M$. This $h$ is called a compatible collaring. For $Y$ a manifold the result is due to Hudson and Zeeman [7]. Also it is shown that pseudo-isotopic imbeddings of a polyhedron $X$ into an (unbounded) manifold $M$ with $\operatorname{dim} M \geqq \operatorname{dim} X+3$ are ambient pseudo-isotopic. (Pseudo-isotopy is often called concordance. For $X$ a manifold this was proved in [6].)

We define an $n$-thickening $\left({ }^{2}\right)$ of a polyhedron $X$ to be an $n$-manifold $T^{n}$ with boundary such that $X \subset$ int $T$ (the interior of $T$ ) and $T$ is P.L. homeomorphic fixing $X$ to a regular neighbourhood of $X$ in int $T$. Two such are equivalent if they are P.L. homeomorphic fixing $X$ pointwise. The set of equivalence classes is denoted $\mathscr{T}^{n}(X)$.

$\S 3$ shows that for any regular homotopy class of immersions of the polyhedron $X$ into an $n$-manifold $M^{n}$ without boundary, $n \geqq \operatorname{dim} X+3$, there is an $n$-thickening $T^{n}$ of $X$ well defined to P.L. isomorphism, fixing $X$, by this condition: any immersion $X \rightarrow M$ in the regular homotopy class extends to an immersion $T \rightarrow M$.

$\S 4$ gives a stability theorem for thickenings. For any polyhedron $X$ the stabilizing map

$$
s_{n}: \mathscr{T}^{n}(X) \rightarrow \mathscr{T}^{n+1}(X)
$$

is defined by crossing thickenings with the interval.

THEOREM 7. $s_{n}$ is surjective for $n \geqq 2 \operatorname{dim} X$ and bijective for $n>2 \operatorname{dim} X$.

(Wall proved a similar result for $X$ compact and $n>5$, using handlebody theory [18].)

As a corollary we find that for $n>2 \operatorname{dim} X$, the elements of $\mathscr{T}^{n}(X)$ are characterized by their stable tangent bundles and all possible stable bundles occur. This means $\mathscr{T}^{n}(X) \cong\left[X, B_{\mathrm{PL}}\right]$. Another corollary is the (known) stability theorem for the block bundles of Rourke-Sanderson.

Our last result is a best possible version of Mazur's stable isomorphism principle for unbounded P.L. manifolds that has hitherto been proved in only a few cases (cf. [15]).

Theorem 8 (Tangential Equivalence Principle). Let $f: M_{1}^{n} \rightarrow M_{2}^{n}$ be a not necessarily proper homotopy equivalence of unbounded P.L. $n$-manifolds, $n \geqq 5$, such that:

(2) We borrow the term from C. T. C. Wall who applies it to a somewhat different entity [17]. 
(1) $f$ is covered by a bundle map of stable tangent (micro)-bundles.

(2) $M_{1}$ and $M_{2}$ have spines of dimension $<n / 2$, i.e., are interiors of $n$-thickenings of complexes of dimension $<n / 2$.

Then $f$ is homotopic to a P.L. homeomorphism-the homotopy being perhaps not proper.

This result genuinely belongs to the category of all (not just proper) P.L. maps. If stated for proper maps it is false (cf. example in [15, \$2]).

The restriction $n \geqq 5$ is almost certainly unnecessary. It appears that for $n \leqq 4$ the result could be proved using handlebody theory with handles of dimensions 0 and 1. The other dimension restrictions for both theorems of $\S 4$ are of course familiar from vector bundle stability. It costs us considerable effort to do so well in this general setting. We give easy counterexamples to show one cannot do better.

$\S 5$ establishes or indicates how to establish relative versions of the above results. $\S 6$ indicates how to translate them into the category of differentiable manifolds, even in relative form.

1. Regular neighbourhoods of $X \times I$. The aim of this section is to prove a "tool" theorem (Theorem 1) which can be regarded as one of the main results of the paper. Recall that the whole discussion is in the P.L. (= piecewise linear) category with the few conventions mentioned in the introduction.

Let $X$ be a (possibly noncompact) polyhedron, $I$ the unit interval $[0,1]$, and suppose that $X \times I$ be imbedded as a closed subpolyhedron in a manifold $M$ in such a way that $(X \times I) \cap \partial M=X \times\{0,1\}$ (note that $X \times I$ is to be regarded as a subset of $M)$. Let $P$ be a regular neighbourhood of $X \times I$ in $M$, meeting $\partial M$ regularly. Thus $P$ meets $\partial M$ in the disjoint union $P_{0} \cup P_{1}$ of a regular neighbourhood $P_{0}$ of $X \times 0$ in $\partial M$ and a regular neighbourhood $P_{1}$ of $X \times 1$ in $\partial M$.

THEOREM 1. If $n=\operatorname{dim} M \geqq \operatorname{dim}(X \times I)+3$, there exists a homeomorphism $f: P_{0} \times I \rightarrow P$ such that:

(i) For all $x \in X$ and $t \in I, f((x, 0), t)=(x, t) \in X \times I \subset P$.

(ii) $f\left(P_{0} \times 0\right)=P_{0}$ and $f\left(P_{0} \times 1\right)=P_{1}$.

REMARK 1. In a sense, (ii) adds nothing to the theorem. If $f$ satisfies only (i), an application of the regular neighbourhood uniqueness theorem permits one to change $f$ so that it satisfies (i) and (ii) (this is explained in the proof below).

REMARK 2. The restriction $n \geqq \operatorname{dim}(X \times I)+3$ is clearly necessary. For example, let $\left(S^{n}, K^{n-2}\right)$ be a knot of $S^{n-2} \cong K^{n-2}$ in $S^{n}$ which is locally knotted (e.g., the suspension of a knotted pair of one lower dimension). Let $p$ and $q$ be two points of $K^{n-2}$ at which $K^{n-2}$ is locally unknotted in $S^{n}$. Excising small neighbourhoods of $p$ and $q$ gives a pair $\left(M^{n}, X \times I\right)$ in which $X \times 0$ and $X \times 1$ are $(n-3)$-spheres imbedded locally flatly in $\partial M$. If the theorem were applicable, $X \times I$ would not be locally knotted in $M$. 
Proof of Theorem 1. The procedure of the proof is to reduce the result to an assertion that a particular isotopy can be extended to an ambient isotopy, and then to establish that assertion.

Let $M^{+}$be the union of $M$ and $\partial M \times[-1,1]$ with $\partial M$ identified with $\partial M \times 1$ in the natural way. Set $J=[-2,2] \supset I$, and extend the inclusion $X \times I \rightarrow M$ to the unique imbedding $e$ of $X \times J$ into $M^{+}$such that

$$
e(X \times J)=((X \times 0) \times[-1,1]) \cup(X \times I) \cup((X \times 1) \times[-1,1]) \subset M^{+},
$$

and such that, for each of the three parts of the image, $e$ has the identity component on $X$ and is linear on the respective interval factor. Let $h: J \rightarrow J$ be the homeomorphism defined by $h \mid \partial J=$ identity, $h(-1)=0, h(0)=1$ and extending linearly.

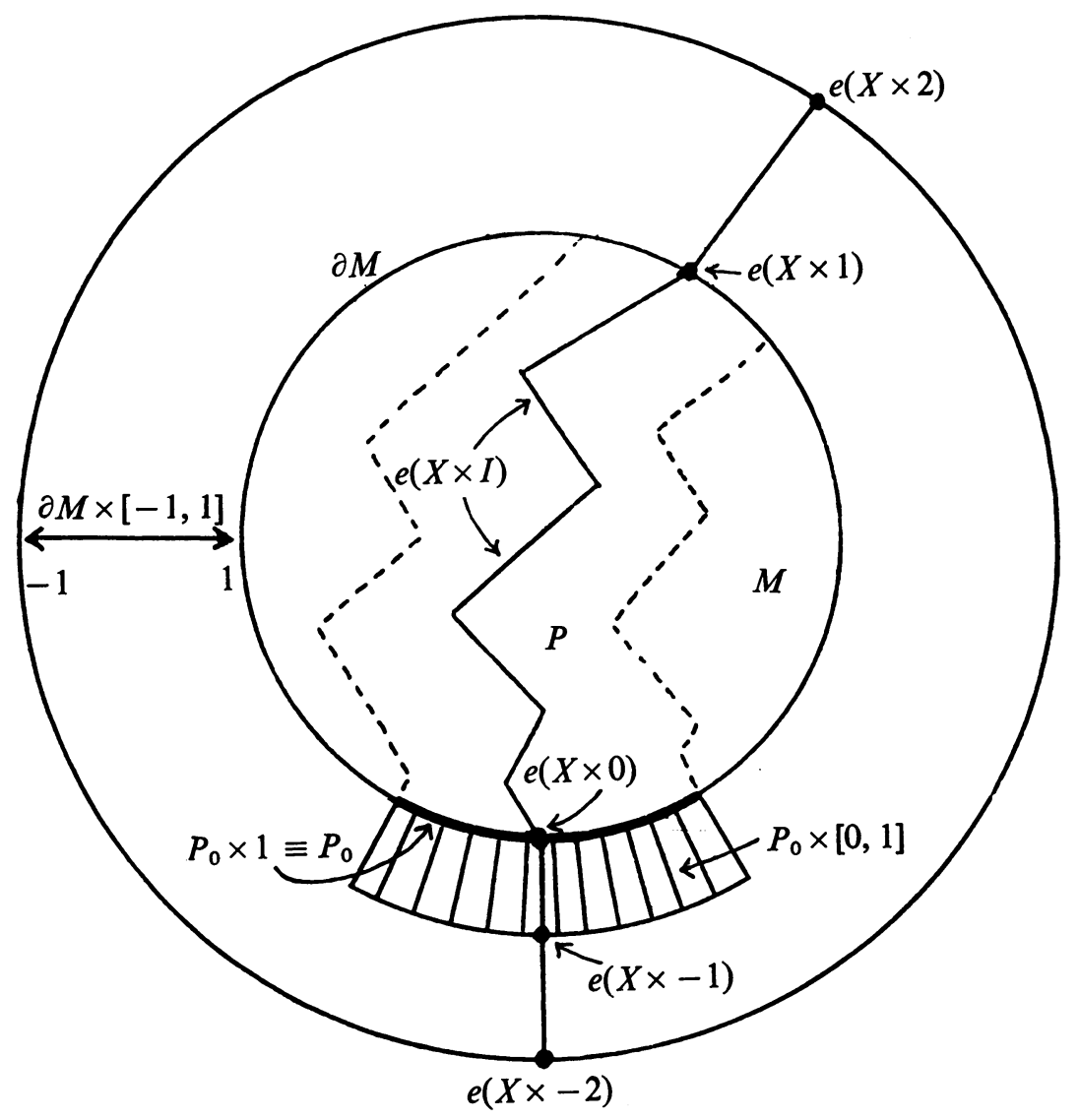

The following assertion will be proved later:

ASSERTION A. The homeomorphism $1_{X} \times h: X \times J \rightarrow X \times J$ extends to a homeomorphism of $M^{+}$; i.e., there exists a homeomorphism $H: M^{+} \rightarrow M^{+}$such that $e\left(1_{x} \times h\right)=H e$.

Now, $1_{X} \times h$ maps $X \times[-1,0]$ to $X \times[0,1]=X \times I$. Hence $H$ maps the regular neighbourhood $P_{0} \times[0,1]$ of $e(X \times[-1,0])$ modulo $e(X \times\{-1,0\})$ in $M^{+}$to some 
regular neighbourhood $H\left(P_{0} \times[0,1]\right)$ of $e(X \times I)=X \times I$ modulo $X \times \partial I$ in $M^{+}$. But $P$ is another such neighbourhood of $X \times I$ in $M^{+}$and the uniqueness theorem for relative regular neighbourhoods [1] guarantees the existence of a further homeomorphism $\theta: M^{+} \rightarrow M^{+}$, fixed on $X \times I$ such that $\theta H\left(P_{0} \times I\right)=P$. Then $f^{\prime}=\theta H$ clearly satisfies condition (i) of the theorem.

$f^{\prime}$ can now be altered to produce an $f$ satisfying both conditions. For the uniqueness theorem for regular neighbourhoods applied to the two regular neighbourhoods, $f^{\prime}\left(P_{0} \times \partial I\right)$ and $P_{0} \cup P_{1}$, of $X \times \partial I$ in $\partial P$ provides an isotopy $\phi: \partial P \times I$ $\rightarrow \partial P \times I$ such that $\phi_{1}\left(f^{\prime}\left(P_{0} \times \partial I\right)\right)=P_{0} \cup P_{1}$ (where $\phi_{1}: \partial P \rightarrow \partial P$ is given by $\left.\phi_{1}(p)=\phi(p, 1)\right)$ and $\phi$ is the identity on $(\partial P \times 0) \cup(N \times I)$ where $N$ is some regular neighbourhood of $X \times \partial I$ in $\partial P$. Let $c: \partial P \times I \rightarrow P$ be an imbedding collaring $\partial P$ so that for $p \in \partial P, c(p, 1)=p$ and so that $c((\partial P-N) \times I) \cap(X \times I)=\varnothing$. Then define $\psi: P \rightarrow P$ by $\psi \mid(P-c(\partial P \times I))=$ identity, and $\psi \mid c(\partial P \times I)=c \phi c^{-1}$; let $f=\psi f^{\prime}$, and it is clear that $f$ satisfies both (i) and (ii).

Theorem 1 has now been reduced to Assertion A, and the proof of the latter follows at once from two lemmas presented below.

Definition. A cone family in a polyhedron $X$ is a locally finite, possibly infinite, family $\mathscr{Y}=\left\{Y_{1}, Y_{2}, Y_{3}, \ldots\right\}$ of compact P.L. cones $Y_{i} \cong a_{i} Z_{i}$ contained in $X$ such that:

(i) The topological frontier in $X$ of each cone $Y_{i}$ is a subset of its base $Z_{i}$.

(ii) The open cones $Y_{i}^{-}=a_{i} Z_{i}-Z_{i}$ are mutually disjoint.

Definition. A homeomorphism, $h: X \rightarrow X$, of the polyhedron $X$ is cone supported on the cone family $\mathscr{Y}=\left\{Y_{i} \mid i=1,2,3, \ldots\right\}$ if $h \mid\left(X-\bigcup_{i} Y_{i}^{-}\right)$is the identity and $h\left(Y_{i}\right)=Y_{i}$ for each $i$.

If $X$ underlies a simplicial complex $K$ of dimension $k$, then for each dimension $t$, $0 \leqq t \leqq k$, there is a cone family $\mathscr{Y}^{t}$ which can be defined as follows. $\mathscr{Y}^{t}$ contains one cone $Y_{\sigma}$ for each $t$-simplex $\sigma$ of $K$, where $Y_{\sigma}$ underlies $\operatorname{star}\left(\sigma, K_{t}\right)$ where $K_{t}$ is the first barycentric derived of $K$ modulo the $t$-skeleton of $K$ (i.e., $K_{t}$ is obtained from $K$ by subdividing barycentrically the simplexes of $K$ of dimension greater than $t$ in some order of increasing dimension). Now if $\hat{\sigma}$ is the barycentre of $\sigma, \operatorname{star}\left(\sigma, K_{t}\right)$ can be regarded as a cone with vertex $\hat{\sigma}$ and base $\partial \sigma *$ link $\left(\sigma, K_{t}\right)$ and hence $Y_{\sigma}$ inherits a cone structure.

Lemma 1. Let $K$ be a complex and $H: K \times I \rightarrow K \times I$ be a P.L. homeomorphism fixed on $K \times \partial I$ such that $H(\sigma \times I)=\sigma \times I$ for each simplex $\sigma \in K$. Then $H$ can be expressed as a product of finitely many cone-supported homeomorphisms of $K \times I$ to itself such that for each cone $Y \cong a Z$ in the supporting families, $Y \cap(K \times \partial I)$ is contained in the base $Z$ of $Y$.

Proof. Suppose inductively that a P.L. homeomorphism $h_{r}: K \times I \rightarrow K \times I$ has been defined such that:

(i) $h_{r} \mid K \times \partial I=1$.

(ii) $h_{r}(\sigma \times I)=\sigma \times I$ for all simplexes $\sigma \in K$. 
(iii) $h_{r} \mid K^{r} \times I=H$ where $K^{r}$ denotes the $r$-skeleton of $K$.

(iv) $h_{r}$ is a finite product of homeomorphisms supported on families of cones of the required type.

The induction begins with $h_{-1}=1$ (and $K^{-1}=\varnothing$ ). The induction step is as follows. Let $f$ be the homeomorphism of $K^{r+1} \times I$ given by $f=H h_{r}^{-1} \mid K^{r+1} \times I$. Note that $f \mid K^{r} \times I=1$. For each $(r+1)$-simplex $\sigma \in K$ define $\bar{f}_{\sigma}$, a homeomorphism of $\operatorname{star}\left(\sigma, K_{r+1}\right) \times I$, such that $\bar{f}_{\sigma}$ is an extension of $f \mid \sigma \times I$ and

$$
\bar{f}_{\sigma} \mid\left(\partial \sigma * \operatorname{link}\left(\sigma, K_{r+1}\right)\right) \times I \cup \operatorname{star}\left(\sigma, K_{r+1}\right) \times \partial I=1 .
$$

(Recall that $K_{r+1}$ is the first derived of $K$ modulo $K^{r+1}$, see above.) If $\tau_{1}, \tau_{2}, \ldots, \tau_{m}$ are the simplexes of $\operatorname{star}\left(\sigma, K_{r+1}\right)$ with $\sigma \prec \tau_{j}$, taken in some order of increasing dimension, $\bar{f}_{\sigma}$ can be defined inductively on $j$ to give homeomorphisms of $\tau_{j} \times I$. At each stage it is only necessary to extend a homeomorphism of the boundary of the ball $\tau_{j} \times I$ to its interior. Thus $\bar{f}_{\sigma}$ can be defined, and have the additional property that for each $\tau \in \operatorname{star}\left(\sigma, K_{r+1}\right), \bar{f}_{\sigma}(\tau \times I)=\tau \times I$.

Now, using the cone family $\mathscr{Y}^{r+1}=\left\{Y_{\sigma}: \sigma\right.$ is a $(r+1)$-simplex of $\left.K\right\}$ note that $Y_{\sigma} \times I$ is a cone with vertex $(\hat{\sigma}, 1 / 2)$ and base equal to $\left(\partial \sigma * \operatorname{link}\left(\sigma, K_{r+1}\right)\right) \times I$ $\cup \operatorname{star}\left(\sigma, K_{r+1}\right) \times \partial I$. Hence define a homeomorphism $\bar{f}: K \times I \rightarrow K \times I$ by $\bar{f} \mid Y_{\sigma} \times I=\bar{f}_{\sigma}$ for each $(r+1)$-simplex $\sigma$ of $K$. Then $\bar{f}$ is cone supported on the cone family $\mathscr{Y}^{r+1} \times I=\left\{Y_{\sigma} \times I\right\}, \bar{f}(\rho \times I)=\rho \times I$ for all $\rho \in K$, and $\bar{f} \mid K^{r+1} \times I=f=$ $H h_{r}^{-1} \mid K^{r+1} \times I$. Finally, define $h_{r+1}=\bar{f} h_{r}$, and it is immediate that $h_{r+1}$ has the required properties.

Reference [10] discusses P.L. imbeddings $f$ of a compact cone $a Z$ into an $n$-ball $B^{n}$ such that $f^{-1}\left(\partial B^{n}\right)=Z$. The main conclusion (called the Cone Unknotting Theorem) states that, if $n-\operatorname{dim}(a Z) \geqq 3$, two such imbeddings that agree on $Z$ are isotopic by an ambient isotopy which keeps $\partial B^{n}$ fixed. The next lemma is an easy consequence of this fact.

LemMa 2. Let $X$ be a polyhedron embedded in a manifold $M$ such that $\operatorname{dim} M$ $-\operatorname{dim} X \geqq 3$. Let $h: X \rightarrow X$ be a homeomorphism cone-supported on a cone-family $\mathscr{Y}=\left\{Y_{i}=a_{i} Z_{i}: i=1,2,3, \ldots\right\}$ in $X$ such that for each $i, Y_{i} \cap \partial M \subset Z_{i}$. Then there is a homeomorphism $H: M \rightarrow M$ such that $H \mid X=h$. In fact $H$ is isotopic to $1_{M}$ keeping $\partial M$ fixed.

Proof. Let $B$ be a regular neighbourhood in $M$ of $\bigcup_{i} Y_{i}$ modulo $\bigcup_{i} Z_{i}$. Then $B$ is a union of regular neighbourhoods, $B_{i}$, in $M$ of $Y_{i}$ modulo $Z_{i}$. Each $B_{i}$ is a ball of dimension $n=\operatorname{dim} M$, and the assumption $Y_{i} \cap \partial M \subset Z_{i}$ guarantees that $Y_{i} \cap \partial B$ is exactly $Z_{i}$. By construction the $B_{i}$ have disjoint interiors. The lemma is now an immediate consequence of the Cone Unknotting Theorem [10], mentioned above, applied to each cone $Y_{i} \subset B_{i}$.

Proof of Assertion A. Let $g=e\left(1_{X} \times h\right) e^{-1}: e(X \times J) \rightarrow e(X \times J)$.

By Lemma 1, $g$ is a finite composition of cone-supported homeomorphisms such that each cone in the supporting families meets $\partial M^{+}$in a subset of its base. Now 
finitely many applications of Lemma 2 imply that $g$ extends to a homeomorphism $H: M^{+} \rightarrow M^{+}$. Thus $H e=e\left(1_{X} \times h\right)$ as required.

The proof of Theorem 1 is now complete.

2. Pseudo-isotopy and ambient pseudo-isotopy. This section establishes two results which follow easily from the previous section; they will not, however, be used in the remainder of this paper.

Let $Q$ be a P.L. manifold, let $e: X \times I \rightarrow Q \times I$ be a P.L. imbedding where $X$ is a polyhedron such that $e^{-1}(\partial(Q \times I))=X \times \partial I$ and $e(X \times t) \subset$ int $Q \times t$ for $t=0,1$. Then $e$ gives what is called a pseudo-isotopy (or concordance) of imbeddings into $Q$ from $e_{0}$ to $e_{1}$ where $e(x, t)=\left(e_{t}(x), t\right)$ for $t=0,1$.

THEOREM 2. If $\operatorname{dim} Q \geqq \operatorname{dim} X+3$, then there exists a homeomorphism $f: Q \times I$ $\rightarrow Q \times I$ such that $f \mid Q \times 0$ is the identity, and for all $(x, t) \in X \times I, f\left(e_{0}(x), t\right)=e(x, t)$. (Thus $f$ is an ambient pseudo-isotopy which covers $e$.)

Proof. Firstly note that $e(X \times I)$ collapses to $e(X \times 0)$ and that $Q \times I$ collapses to $Q \times 0$. It follows at once from [5] that $Q \times I$ collapses to $(Q \times 0) \cup e(X \times I)\left({ }^{3}\right)$. Hence, by a criterion of Hudson and Zeeman [8], $Q \times 1$ is a regular neighbourhood of $(Q \times 0) \cup e(X \times I)$ modulo $e(X \times 1)$ in $Q \times[0,2]$.

Extend $e$ to an imbedding $e: X \times[-2,2] \rightarrow Q \times[-2,2]$ by defining

$$
\begin{aligned}
e(x, t) & =\left(e_{0}(x), t\right) \text { for } t \leqq 0, \\
& =\left(e_{1}(x), t\right) \text { for } t \geqq 1 .
\end{aligned}
$$

Define a homeomorphism $h:[-2,2] \rightarrow[-2,2]$ by $h( \pm 2)= \pm 2, h(-1)=0$, $h(0)=1$ and extending linearly.

Theorem 1 states that any regular neighbourhood $P$ of $e(X \times[-2,2])$ in $Q \times[-2,2]$, meeting the boundary regularly, admits the structure of a product with $[-2,2]$. Using this, together with a collar of the frontier of $P$ in $Q \times[-2,2]-P^{\circ}$, $e\left(1_{X} \times h\right) e^{-1}$ extends to a P.L. homeomorphism $H$ of $Q \times[-2,2]$. Thus $H e$ $=e\left(1_{X} \times h\right)$ (alternatively, $H$ may be constructed as in the proof of Assertion A), and $H$ may be taken to be the identity on $\partial(Q \times[-2,2])$.

Now, by applying the relative regular neighbourhood uniqueness theorem [1] to neighbourhoods in $Q \times[-2,2]$ of $(Q \times-2) \cup e(X \times[-2,0])$ modulo $e(X \times[0,2]), H$ can be adjusted (keeping $e(X \times[-2,2])$ fixed) so that

$$
H(Q \times[-2,-1])=Q \times[-2,0] .
$$

Then $H(Q \times[-1,0])$ is a regular neighbourhood of $(Q \times 0) \cup e(X \times I)$ modulo

$\left({ }^{3}\right)$ The idea of collapsing of noncompact polyhedra is discussed in [16]. In [5] Hudson proves, via the idea of sunny collapsing, that if $M$ is a P.L. manifold with subpolyhedra $X$ and $Y$, then $M \searrow Y$ and $X \searrow Y$ implies that $M \searrow X$ provided that $\operatorname{dim} M-\operatorname{dim}(X-Y) \geqq 3$. 
$e(X \times[1,2])$ in $Q \times[0,2]$; as mentioned above, $Q \times[0,1]$ is another such neighbourhood. Thus using uniqueness again, $H$ can be further adjusted so that

$$
H(Q \times[-1,0])=Q \times[0,1] .
$$

Define $f^{\prime}: Q \times I \rightarrow Q \times I$ by $f^{\prime}(q, t)=H(q, t-1)$ then $f^{\prime}$ is a homeomorphism, $f^{\prime}(Q \times t)=Q \times t$ for $t=0,1$, and

$$
\begin{aligned}
f^{\prime}\left(e_{0}(x), t\right) & =H\left(e_{0}(x), t-1\right) \\
& =H(e(x, t-1)) \\
& =e\left(1_{X} \times h\right)(x, t-1) \\
& =e(x, t) .
\end{aligned}
$$

Thus $f^{\prime}$ has the required properties for $f$, with the exception of $f^{\prime}$ being the identity on $Q \times 0$. But if $\phi: Q \rightarrow Q$ is the homeomorphism defined by $f^{\prime}(\phi(q), 0)=(q, 0)$ for all $q \in Q, \phi$ is the identity on $e_{0} X$, so that $f$ can be defined by $f=f^{\prime}\left(\phi \times 1_{I}\right)$.

Theorem 3 (Compatible Collars). Let $W$ be a P.L. $(n+1)$-manifold and $V$ be an $n$-submanifold of $\partial W$. Let $X$ be a polyhedron, and let $e: X \times I \rightarrow W$ be an imbedding so that $e(X \times I) \cap \partial W=e(X \times 0) \subset$ int $V$. Suppose $\operatorname{dim} X+3 \leqq n$, then there exists a P.L. imbedding $f: V \times I \rightarrow W$ such that $f(V \times I)$ is a neighbourhood of $V$ in $W$, $f(v, 0)=v$ for all $v \in V$ and $f(e(x, 0), t)=e(x, t)$.

Proof. A regular neighbourhood in $W$ of $V \cup e(X \times I)$ modulo $e(X \times 1)$ is also a regular neighbourhood of $V$ in $W$ since there is a collapsible retraction from $V \cup(X \times I)$ to $V$ [1]. Hence this regular neighbourhood can be identified with $V \times I$, with $V$ corresponding to $V \times 0$. An application of Theorem 2 (with $V$ in place of $Q$ ) then immediately gives the required result.

3. Regular neighbourhoods and immersions of polyhedra. If $X$ is a subpolyhedron of a polyhedron $Y$, the idea of a regular neighbourhood of $X$ in $Y$ is discussed in [19]. If $X$ is a polyhedron a regular neighbourhood of $X$ (sometimes referred to as an "abstract" regular neighbourhood) is a pair $(e, R(X))$ where $R(X)$ is a polyhedron, $e: X \rightarrow R(X)$ is an imbedding, and $R(X)$ is a regular neighbourhood of $X$ in $R(X)$. An immersion of $X$ in another polyhedron $Y$ is simply a P.L. map $X \rightarrow Y$ which is locally an imbedding. It will be shown below that, with any immersion $i: X \rightarrow Y$, there is associated a regular neighbourhood $R_{i}(X)$ in a natural and essentially unique way. If $i$ happens to be an imbedding then $R_{i}(X)$ can be identified with a regular neighbourhood of $i(X)$ in $Y$, and $e: X \rightarrow R_{i}(X)$ identifies with $i$. Thus this theory is but a generalization of the idea of regular neighbourhood in the context of immersions, and, in the case when $X$ and $Y$ are manifolds without boundary, the idea has already been explored in [2]. One of the chief results of this section is that if $Y$ is a manifold without boundary, then $R_{i}(X)$ depends only on the (pseudo) regular homotopy class of $i$. 
Definition. Let $X$ and $Y$ be polyhedra, $i: X \rightarrow Y$ be an immersion. A regular neighbourhood of $X$ associated with $i$ is a regular neighbourhood $\left(e, R_{i}(X)\right)$ of $X$ together with an immersion $j: R_{i}(X) \rightarrow Y$ such that the diagram

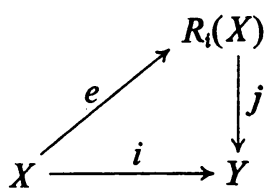

commutes, and for each $x \in X$ there is a neighbourhood $N$ of $e(x)$ in $R_{i}(X)$ such that $j \mid N$ is an imbedding and $j(N)$ is a neighbourhood of $i(x)$ in $Y$.

Note that the existence of one such neighbourhood $N$ for $x$ implies the existence of arbitrarily small such neighbourhoods.

THEOREM 4. If $i: X \rightarrow Y$ is an immersion of the polyhedron $X$ in the polyhedron $Y$, then there exists a regular neighbourhood of $X$ associated with $i$.

Proof. There are triangulations of $X$ and $Y$, which, by abuse of notation, will also be denoted by $X$ and $Y$, with respect to which $i: X \rightarrow Y$ is simplicial. Thus $i$ imbeds the star of each simplex of $X$. Construct a new complex $R$ as follows. Corresponding to each simplex $\tau \in X$ and each $\sigma \in Y$ such that $\sigma \succ i \tau$, take a (closed) simplex $\sigma_{\tau}$ and a simplicial isomorphism $j_{\tau}: \sigma_{\tau} \rightarrow \sigma$. Let $R$ be the union of all such simplexes $\sigma_{\tau}$ with $X$, with $\left(j_{\tau}\right)^{-1}(i \tau)$ identified with $\tau$ by the homeomorphism $i^{-1} j_{\tau}$. The union of the various $j_{\tau}$ defines a simplicial map $j: R \rightarrow Y$ such that $\hat{j}$ imbeds each simplex of $\hat{K}$, and $\hat{j} \mid X=i$. Now if $\tau \in X, \rho_{1}$ and $\rho_{2} \in R, \rho_{1} \succ \tau$, and $\rho_{2} \succ \tau$, and $\hat{j} \rho_{1}=j \rho_{2}$, identify $\rho_{1}$ and $\rho_{2}$ by the homeomorphism $\left(\hat{j} \mid \rho_{2}\right)^{-1}\left(\hat{j} \mid \rho_{1}\right)$. There results a complex $K$ and a map $j: K \rightarrow Y$ induced by $\hat{j}$.

Now $X \subset K$ and, if $\tau \in X$, by construction $j$ imbeds $\operatorname{star}(\tau, K)$ onto $\operatorname{star}(i \tau, Y)$. Let $R_{i}(X)$ be a second derived neighbourhood of $X$ in $K$, i.e., $R_{i}(X)=N\left(X, K^{\prime \prime}\right)$. Then $R_{i}(X)$ is a regular neighbourhood of $X$ in $K$, and hence also $R_{i}(X)$ is a regular neighbourhood of $X$ in $R_{i}(X)$. Further, $j \mid X=i$; as $j \mid \operatorname{star}(\tau, K)$ is an imbedding for each $\tau \in K, j \mid R_{i}(X)$ is certainly an immersion; and if $x$ is a point of $X$, $j\left(N\left(x, K^{\prime \prime}\right)\right)=N\left(i x, Y^{\prime \prime}\right)$. Thus, defining $e: X \rightarrow R_{i}(X)$ to be the inclusion, $\left(e, R_{i}(x), j\right)$ satisfies all the conditions of a regular neighbourhood associated with $i$.

Theorem 4 has established the existence of regular neighbourhoods associated with an immersion, and the next theorem and its corollary establish uniqueness. It is useful to record first an elementary result:

LEMMA 3. Let $f: X \rightarrow Y$ be an immersion of polyhedra, let $W$ be connected and $\phi, \psi: W \rightarrow X$ be maps such that $f \phi=f \psi$ and, for some $w \in W, \phi(w)=\psi(w)$, then $\phi=\psi$.

Proof. Let $S=\{s \in W: \phi(s)=\psi(s)\} ; w \in S$ so $S \neq \varnothing$. The continuity of $\phi$ and $\psi$ implies that $S$ is closed, and the local injectivity of $f$ shows that $S$ is open. Then $S=W$ since $W$ is connected. 
THEOREM 5. Let $X$ and $Y$ be polyhedra and $i: X \rightarrow Y$ be an immersion. Let $X \stackrel{e_{1}}{\rightarrow} R_{1} \stackrel{j_{1}}{\rightarrow} Y$ and $X \stackrel{e_{2}}{\rightarrow} R_{2} \stackrel{j_{2}}{\rightarrow} Y$ be two regular neighbourhoods of $X$ associated with $i$. Then there exists a regular neighbourhood $R_{1}^{\prime}$ of $e_{1}(X)$ in $R_{1}$, and a P.L. imbedding $h$ which makes the following diagram commutative.

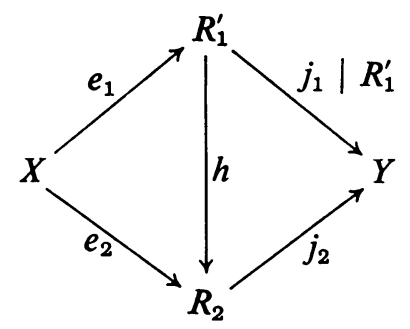

Proof. From the definition of a regular neighbourhood associated with $i$, for each $x \in X$ there are neighbourhoods $N_{1}$ and $N_{2}$ of $e_{1}(x)$ in $R_{1}$ and of $e_{2}(x)$ in $R_{2}$ such that $j_{1} \mid N_{1}$ and $j_{2} \mid N_{2}$ are imbeddings onto the same neighbourhoods of $i(x)$ in $Y$. Thus $j_{1}^{-1} j_{2}: N_{2} \rightarrow N_{1}$ is a homeomorphism such that $j_{1}^{-1} j_{2} e_{2} \mid e_{2}^{-1} N_{2}$ $=e_{1} \mid e_{1}^{-1} N_{1}$. Now let $\left\{N_{2}^{\alpha}\right\}_{\alpha \in A}$ be a collection of open neighbourhoods of points $e_{2}\left(x_{\alpha}\right)$ in $R_{2}$ such that $e_{2} X$ is covered by the $N_{2}^{\alpha}$, for each $N_{2}^{\alpha}$ there is an $N_{1}^{\alpha}$ with the above properties, and any pair of the neighbourhoods $\left\{N_{2}^{\alpha}\right\}$ intersect in a connected neighbourhood of some point of $e_{2} X$ (e.g., $\left\{N_{2}^{\alpha}\right\}$ can be taken to be the open star neighbourhoods of the vertices of $e_{2} X$ in some sufficiently fine triangulation). Each map $j_{1}^{-1} j_{2} \mid N_{2}^{\alpha}: N_{2}^{\alpha} \rightarrow R_{1}$ is defined and is an imbedding; furthermore, since $j_{1}$ is an immersion, by Lemma 3 if two of the $N_{2}^{\alpha}$ 's intersect, the two maps agree on the intersection (the intersection is connected and the maps agree on some point of $e_{2} X$ in the intersection). Let $U_{2}=\bigcup_{\alpha \in A} N_{2}^{\alpha}$, and define $g: U_{2} \rightarrow R_{1}$ by $g\left|N_{2}^{\alpha}=j_{1}^{-1} j_{2}\right| N_{2}^{\alpha}$, then $g$ is a well-defined immersion, $j_{1} g=j_{2}, g e_{2}=e_{1}$, and $g\left(U_{2}\right)$ is a neighbourhood of $e_{1} X$ in $R_{1}$.

In an exactly similar way, there is a neighbourhood $U_{1}$ of $e_{1} X$ in $R_{1}$, with $U_{1} \subset g\left(U_{2}\right)$, and an immersion $f: U_{1} \rightarrow R_{2}$ such that $f U_{1} \subset U_{2}, j_{2} f=j_{1}, f e_{1}=e_{2}$, and $f U_{1}$ is a neighbourhood of $e_{2} X$. It may be assumed that each component of $U_{1}$ intersects $e_{1} X$. Then $j_{1} g f=j_{2} f=j_{1} \mid U_{1}$ and $g f \mid e_{1} X$ is the identity; thus, Lemma 3, applied to each component of $U_{1}$, implies that $g f$ is the inclusion of $U_{1}$ in $R_{1}$. Thus $f$ is injective, and hence, as $f$ is P.L., $f$ is an imbedding. Now let $R_{1}^{\prime}$ be a regular neighbourhood of $e_{1} X$ in $R_{1}$, with $R_{1}^{\prime} \subset U_{1}$, and let $h=f \mid R_{1}^{\prime}$.

COROLlaRY. There exists a homeomorphism $H: R_{1} \rightarrow R_{2}$ such that $\mathrm{He}_{1}=e_{2}$, and $j_{2} H$ is regularly homotopic to $j_{1}$ rel $e_{1} X$.

Proof. Regular neighbourhood uniqueness implies that there exists a homeomorphism $\phi: R_{1} \rightarrow R_{1}^{\prime}$ fixing $e_{1}(X) . h R_{1}^{\prime}$ is a regular neighbourhood of $e_{2} X$ in $R_{2}$ so there is also a homeomorphism $\psi: h R_{1}^{\prime} \rightarrow R_{2}$ fixing $e_{2} X$. Let $H=\psi h \phi$. Using the fact that $\phi$ and $\psi$ are isotopic to the identity maps, it is easy to construct the regular homotopy (see definition below). 
Definition. A P.L. homotopy $f: X \times I \rightarrow Y$ between two immersions of a polyhedron $X$ in a polyhedron $Y$ is a regular homotopy if the associated map $\hat{f}: X \times I \rightarrow Y \times I$, defined by $\hat{f}(x, t)=(f(x, t), t)$, is an immersion. $f$ is a pseudo regular homotopy if there is some P.L. immersion $F: X \times I \rightarrow Y \times I$ with $F^{-1}(Y \times 0)$ $=X \times 0$ and $F^{-1}(Y \times 1)=X \times 1$ such that $f=p_{1} F$ where $p_{1}: Y \times I \rightarrow Y$ is the natural projection.

The definition of a regular neighbourhood associated with an immersion, and the existence and uniqueness theorems lead at once to a few straightforward results which will be needed later in the paper, and it is convenient to list them here.

Property 1. If $i: X \rightarrow Y$ is an imbedding, $X \stackrel{i}{\rightarrow} R_{i}(X) \stackrel{j}{\rightarrow} Y$ is a regular neighbourhood of $X$ associated with $i$ where $R_{i}(X)$ is a regular neighbourhood of $i(X)$ in $Y$ and $j$ is the inclusion.

Property 2. Let $i: X \rightarrow Y$ be an immersion with associated regular neighbourhood $X \stackrel{e}{\rightarrow} R_{\imath}(X) \stackrel{j}{\rightarrow} Y$. Let $\phi: Y \rightarrow Y \times[-1,1]$ be the imbedding given by $\phi(y)=(y, 0)$ for all $y \in Y$, and let $\psi: R_{i}(X) \rightarrow R_{i}(X) \times[-1,1]$ be defined similarly. Then $X \stackrel{\psi e}{\longrightarrow} R_{i}(X) \times[-1,1] \stackrel{j \times 1}{\longrightarrow} Y \times[-1,1]$ is a regular neighbourhood of $X$ associated with $\phi i$.

Property 3. Let $M$ be a manifold, $X$ a polyhedron and let $i: X \rightarrow M$ be an immersion with associated regular neighbourhood $X \stackrel{e}{\rightarrow} R_{i}(X) \stackrel{j}{\rightarrow} M$, then $R_{i}(X)$ is a manifold and

$$
e^{-1}\left(\partial R_{i}(X)\right)=i^{-1}(\partial M) .
$$

This is so because, from the definition, some neighbourhood of $e(X)$ in $R_{i}(X)$ is a manifold, and therefore a small enough regular neighbourhood of $e(X)$ in $R_{i}(X)$ is a manifold; thus by uniqueness of regular neighbourhoods in $R_{i}(X), R_{i}(X)$ itself is a manifold.

Property 4. With the notation of Property 3 , there is a regular neighbourhood $V$ of $e\left(i^{-1} \partial M\right)$ in $\partial R_{i}(X)$ such that

$$
i^{-1} \partial M \stackrel{e}{\longrightarrow} V \stackrel{j \mid V}{\longrightarrow} \partial M
$$

is a regular neighbourhood of $i^{-1} \partial M$ associated with the immersion

$$
i \mid i^{-1} \partial M: i^{-1} \partial M \rightarrow \partial M .
$$

This follows from the fact that $j^{-1} \partial M$ is a neighbourhood of $i^{-1} \partial M$ in $\partial R_{i}(X)$ and so $V$ can be taken so that $V \subset j^{-1} \partial M$.

The next theorem is an application of Theorem 1 to the theory of immersions of polyhedra in manifolds of codimension at least three. It gives a method of showing when different immersions of a polyhedron in a manifold have equivalent associated regular neighbourhoods, and it is the crucial result needed in the next section.

THEOREM 6. Let $i: X \times I \rightarrow M$ be an immersion of the polyhedron $X \times I$ in the P.L. manifold $M$, with $i^{-1} \partial M=X \times \partial I$ and $\operatorname{dim} M-\operatorname{dim}(X \times I) \geqq 3$. Let $i_{t}: X \rightarrow \partial M$ 
be the immersion defined by $i_{t}(x)=i(x, t)$ where $t=0$ or 1 for all $x \in X$, and let $X \stackrel{i_{t}}{\rightarrow} R_{i_{t}}(X) \stackrel{j_{t}}{\rightarrow} \partial M$ be associated regular neighbourhoods. Then there is a homeomorphism $h: R_{i_{0}}(X) \rightarrow R_{i_{1}}(X)$ such that $e_{0}=e_{1}$.

Note. When such an $h$ exists, the two regular neighbourhoods will be called equivalent.

Proof. Let $X \times I \stackrel{e}{\rightarrow} R_{i}(X \times I) \stackrel{j}{\rightarrow} M$ be a regular neighbourhood of $X \times I$ associated with $i$. By Property 4 above, and by the Corollary to Theorem 5 , it may be assumed that $R_{t_{t}}(X)$ is any given regular neighbourhood of $e(X \times t)$ in $\partial\left(R_{i}(X \times I)\right)$, that $e_{t}(x)=e(x, t)$, and that $j_{t}=j \mid R_{i_{t}}(X)$ for $t=0$ or 1 . Now $R_{i}(X)$ is a regular neighbourhood of $e(X \times I)$ in $R_{i}(X)$ and $e^{-1} \partial R_{i}(X)=X \times \partial I$. Thus by Theorem 1, there is a homeomorphism $f: R_{i_{0}}(X) \times I \rightarrow R_{i}(X)$ such that for $x \in X$ and $t \in I, f\left(e_{0} x, t\right)=e(x, t)$, and $f\left(R_{i_{0}}(X) \times 0\right)=R_{i_{0}}$ and $f\left(R_{i_{0}}(X) \times 1\right)=R_{i_{1}}$. Define $h: R_{i_{0}}(X) \rightarrow R_{i_{1}}(X)$ by $h(r)=f(r, 1)$ for all $r \in R_{i_{0}}(X)$.

COROLlary. Let $Q$ be a manifold without boundary, and $i_{0}$ and $i_{1}$ be pseudo regularly homotopic immersions of a polyhedron $X$ in $Q$, where $\operatorname{dim} Q-\operatorname{dim} X \geqq 3$. Let $X \stackrel{e_{t}}{\rightarrow} R_{t} \stackrel{j_{t}}{\rightarrow} Q$ be the associated regular neighbourhoods where $t=0$ or 1 . Then there is a homeomorphism $h: R_{0} \rightarrow R_{1}$ such that he $e_{0}=e_{1}$.

Proof. The fact that $i_{0}$ and $i_{1}$ are pseudo regularly homotopic implies there is an immersion $F: X \times I \rightarrow Q \times I$ which satisfies the conditions of the theorem with $Q \times I$ in place of $M$ such that $F(x, t)=\left(e_{t}(x), t\right)$ for $t=0$ or 1 . The result then follows at once by applying the theorem to $F$.

RemarK. As $Q$ has no boundary, $e_{t}(X) \subset$ int $R_{t}$, and so $R_{t}$ is a thickening of $e_{t}(X)$.

4. Stability of thickenings. Recall from the introduction, that an $n$-thickening of a polyhedron $X$ is an $n$-manifold (with boundary), containing $X$ in its interior, which is a regular neighbourhood of $X$ in itself. Two thickenings $e_{1}: X \hookrightarrow T_{1}$ and $e_{2}: X \hookrightarrow T_{2}$ are called equivalent if there is a (P.L.) homeomorphism $h: T_{1} \hookrightarrow T_{2}$ such that $h e_{1}=e_{2}$. The set of equivalence classes of $n$-thickenings of $X$ is denoted $\mathscr{T}^{n}(X)$. The operation which assigns to an $n$-thickening $e: X \rightarrow T$ the $(n+1)$ thickening $e^{0}: X \hookrightarrow T \times[-1,1]$, where $e^{0}(x)=(e(x), 0)$ for all $x \in X$, induces what will be called the stabilization map $s_{n}: \mathscr{T}^{n}(X) \rightarrow \mathscr{T}^{n+1}(X)$.

The main result of this section is the following.

Theorem 7 (Stability of Thickenings). The stabilization map $s_{n}$ is surjective if $n \geqq 2 \operatorname{dim} X$, and injective if $n>2 \operatorname{dim} X$.

Proof of injectivity. Let $\operatorname{dim} X=k$. It is required to prove that if $e_{1}: X \hookrightarrow T_{1}$ and $e_{2}: X \hookrightarrow T_{2}$ are two $n$-thickenings of $X$, and there is a homeomorphism $H: T_{1} \times[-1,1] \rightarrow T_{2} \times[-1,1]$ satisfying $H e_{1}^{0}=e_{2}^{0}$, then, providing $n>2 k$, the thickenings $T_{1}$ and $T_{2}$ are equivalent. 
The one case $n=3, k=1$ escapes the proof presented below. However, it is known that the first Stiefel-Whitney class gives an isomorphism

$$
\mathscr{T}^{n}\left(X^{1}\right) \stackrel{w_{1}}{\cong} H^{1}\left(X^{1}: Z_{2}\right)
$$

for $n \geqq 3$, and this implies the wanted injectivity. For completeness, a proof of this will be given later as Proposition 1 of the Appendix.

Thus assume that $(n, k) \neq(3,1)$. Let $j_{1}$ and $j_{2}$ be the imbeddings of $X$ in $\partial\left(T_{1} \times[-1,1]\right)$ given by the following compositions:

$$
\begin{aligned}
& j_{1}: X \longrightarrow X \times 1 \longleftrightarrow \partial\left(T_{1} \times[-1,1]\right), \\
& j_{2}: X \longrightarrow X \times 1 \longleftrightarrow \partial\left(T_{2} \times[-1,1]\right) \stackrel{H^{-1}}{\longrightarrow} \partial\left(T_{1} \times[-1,1]\right) .
\end{aligned}
$$

Then clearly $T_{1}$ and $T_{2}$ are equivalent to regular neighbourhoods of $X$ associated with $j_{1}$ and $j_{2}$. Now move $j_{2}$ to $j_{2}^{\prime}$ by an ambient isotopy of $\partial\left(T_{1} \times[-1,1]\right)$ so that $j_{2}^{\prime}(X)$ is in general position with respect to $j_{1}(X)$. As $2 \operatorname{dim} X=2 k<n=$ $\operatorname{dim} \partial\left(T_{1} \times[-1,1]\right), j_{2}^{\prime}$ can be chosen so that $j_{2}^{\prime}(X) \cap j_{1}(X)=\varnothing$. (Note. If $X$ is noncompact general position results follow from applications of Zeeman's general position lemma [19] infinitely many times.) Now $j_{1}$ and $j_{2}^{\prime}$ are homotopic in $T_{1} \times[-1,1]$ as each is homotopic to $e_{1}^{0}$; thus there is a homotopy $F: X \times I \rightarrow$ $T_{1} \times[-1,1]$ between $j_{1}$ and $j_{2}^{\prime}$ and, by general position, it may be assumed that

$$
F^{-1}\left(\partial\left(T_{1} \times[-1,1]\right)\right)=X \times \partial I
$$

and that the singular set of $F$ has dimension $2(k+1)-(n+1)=2 k-n+1 \leqq 0$. Hence $F$ is an immersion, and so by Theorem 6, the regular neighbourhoods of $X$ associated with the imbeddings $j_{1}$ and $j_{2}^{\prime}$ are equivalent thickenings. Thus $T_{1}$ and $T_{2}$ are equivalent.

Proof of surjectivity. Here it is necessary to show that if $e: X \hookrightarrow T$ is an $(n+1)$ thickening of the $k$-dimensional polyhedron $X$, and $n \geqq 2 k$, then $T$ can be written as a product $T=T_{0} \times[-1,1]$ so that $X \hookrightarrow T_{0} \times 0$ and $T_{0} \times 0$ is an $n$-thickening of $X$.

By general position results, $e: X \hookrightarrow T$ is homotopic to a map $f^{\prime}: X \rightarrow T$ such that $e(X) \cap f^{\prime}(X)=\varnothing$. Let $N$ be a regular neighbourhood of $e X$ in int $T$ such that $N \cap f^{\prime} X=\varnothing$. Then $T$-int $N \cong \partial N \times I$ (cf. [19]). Projecting $T$-int $N$ onto $\partial N$ shows that $f$ is homotopic to $f^{\prime \prime}: X \rightarrow T$ where $f^{\prime \prime} X \subset \partial N$. By general position arguments, $f^{\prime \prime}$ is homotopic to a map $f: X \rightarrow T$ where $f X \subset \partial N$, and the singularities of $f$ are of dimension $2 k-\operatorname{dim} \partial N=2 k-n \leqq 0$. Hence $f$ is an immersion of $X$ into $\partial N$.

Let $M=T \times R_{+}$where $R_{+}$is the nonnegative reals, and identify $T$ with $T \times 0$ $\subset \partial M$. Then $e$ and $f$ are homotopic in $M$ by a homotopy $F: X \times I \rightarrow M$ which, after moving to general position, has singular set of dimension $(2 k+2)-(n+2) \leqq 0$ (i.e., $F$ is an immersion), and $F^{-1}(\partial M)=X \times \partial I$. By Theorem 6 , assuming for the moment that $(n, k) \neq(2,1)$ as Theorem 6 requires $(n+2)-(k+1) \geqq 3$, the immersions 
$e$ and $f$ of $X$ in $T$ have equivalent associated regular neighbourhoods. However, that associated with $e$ is clearly equivalent to the thickening $T$ itself. But $f$ immerses $X$ in $\partial N$, and by the collaring theorem there is a neighbourhood of $\partial N$ in $T$ homeomorphic to $\partial N \times[-1,1]$ with $\partial N \times 0$ identified to $\partial N$. Hence by $\S 3$ Property 2, the regular neighbourhood of $X$ associated with $f: X \rightarrow T$ is equivalent to $X \rightarrow R(X) \times[-1,1] \rightarrow \partial N \times[-1,1]$ where $R(X)$ is a regular neighbourhood of $X$ associated with $f: X \rightarrow \partial N$. As $\partial N$ is a manifold without boundary, $R(X)$ is an $n$-thickening of $X$; so letting $T_{0}=R(X)$, the result is proved.

There remains to consider the special case when $(n, k)=(2,1)$. Identify $T$ with $T \times 0 \subset T \times R$, then exactly as before, the immersions $e$ and $f$ of $X$ in $T \times R$ have equivalent associated regular neighbourhoods. That associated with $e$ is $s_{3} T$, and (using $\$ 3$ Property 2 again) that associated with $f$ is the 4-thickening $s_{3} s_{2} R(X)$ where, as before, $R(X)$ is the thickening which is the regular neighbourhood associated with $f: X \rightarrow \partial N$. Now using the fact that $s_{3}$ is injective (Proposition 1, in the Appendix), $T$ is equivalent to $s_{2} R(X)$.

Corollary 1 (Block-BUndle StaBility (KNOWN)). $\pi_{k} B_{\mathrm{PL} \sim(n)} \rightarrow \pi_{k} B_{\mathrm{PL} \sim(n+1)}$ is surjective for $k \leqq n$, and bijective for $k<n$.

Proof. According to [13], $\pi_{k} B_{\mathrm{PL} \sim(n)}=\mathscr{T}^{n+k}\left(S^{k}\right)$.

COROLlaRY 2. If $X$ is a polyhedron and $n>2 \operatorname{dim} X$, then

$$
\mathscr{T}^{n}(X) \cong\left[X, B_{\mathrm{PL}}\right]
$$

where $\left[X, B_{\mathrm{PL}}\right]$ is the set of stable isomorphism classes of P.L. microbundles over $X$.

Proof. There is a natural map $\mathscr{T}^{n}(X) \stackrel{c_{n}}{\longrightarrow}\left[X, B_{\mathrm{PL}}\right]$ arising from the restriction to $X$ of the tangent microbundles of thickenings $T$ of $X$. In view of Theorem 7, it is sufficient to show that the induced map $\mathscr{T}(X)=$ ind $\lim _{n} \mathscr{T}^{n}(X) \stackrel{c}{\rightarrow}\left[X, B_{\mathrm{PL}}\right]$ is bijective.

Surjectivity of $c$ is verified as follows. If $\xi$ is a P.L. microbundle over $X$, let $T_{0}$ be a regular neighbourhood of $X$ in some Euclidean space, and $\eta$ be a microbundle over $T_{0}$ such that $\eta \mid X=\xi$. If $T$ is a regular neighbourhood of $X$ in the total space of $\eta, T$ is a thickening of $X$, and $\tau(T)\left|X=\xi \oplus \tau\left(T_{0}\right)\right| X$ where $\tau$ denotes the tangent microbundle. However $\tau\left(T_{0}\right) \mid X$ is trivial and so $c[T]=[\xi]$.

That $c$ is injective is equivalent to the assertion that two thickenings $e_{1}: X \hookrightarrow T_{1}$ and $e_{2}: X \hookrightarrow T_{2}$ which have the same stable tangent microbundle (when restricted to $X$ ) are necessarily stably equivalent thickenings. This can be proved in the following way. If $D^{s}$ is a P.L. s-ball and $*$ is a point of int $D^{s}$, let $j: T_{1} \rightarrow \operatorname{int}\left(T_{2} \times D^{s}\right)$ for some large $s$, be an imbedding such that $j e_{1}=e_{2}^{*}$ where $e_{2}^{*}: X \rightarrow T_{2} \times D^{s}$ is given by $e_{2}^{*}(x)=\left(e_{2}(x), *\right)$. Since $s$ can be made arbitrarily large, it can be assumed that $j$ has a normal microbundle $\nu$. By a result of Milnor on microbundles, if the bundles are pulled back to $X$,

$$
\tau\left(T_{1}\right) \oplus \nu \cong \tau\left(T_{2} \times D^{s}\right)
$$


Passing to stable classes, it follows that $v$ is stably trivial, and so by increasing $s$ still further, it can be assumed that $v$ is trivial (as a microbundle over $X$ and hence also as one over $T_{1}$ ). Then there exists an imbedding

$$
J: T_{1} \times D^{s} \rightarrow \operatorname{int}\left(T_{2} \times D^{s}\right)
$$

so that $J e_{1}^{*}=e_{2}^{*}$. Thus $J\left(T_{1} \times D^{s}\right)$ is a regular neighbourhood of $e_{2}^{*} X$ in $T_{2} \times D^{s}$, and so by uniqueness of regular neighbourhoods, $e_{1}^{*}: X \hookrightarrow T_{1} \times D^{s}$ and $e_{2}^{*}: X \hookrightarrow T_{2}$ $\times D^{s}$ are equivalent thickenings for some large $s$, as was required.

REMARK. Corollary 2 casts a ray of light on an unsolved $\left({ }^{4}\right)$ problem in dimension 5: If $i: K^{2} \hookrightarrow T^{5}$ is a 5-thickening of a compact contractible 2-complex, is $T^{5}$ necessarily P.L. homeomorphic to the disc $D^{5}$ ? If so, one could for example conclude that if $M^{3}$ is a compact contractible 3-manifold, then $M^{3} \times D^{2}$ is a 5-disc. What the corollary shows is that there is a unique 5-thickening of $K^{2}$, without saying it is a 5-disc. But $T$ is clearly contractible with $\partial T$ simply connected. Hence $\partial T$ is a homotopy 4-sphere. Thus the correspondence $K^{2} \leadsto \partial T$ gives a well-defined map from compact contractible 2-complexes to P.L. homeomorphism classes of homotopy 4-spheres. The problem amounts to showing that the map is zero, for if $\partial T$ is a 4-sphere, $T^{5}$ is a 5-disc [11].

The next result, which depends on Theorem 7 , finds the edge of stability in the stable isomorphism theorem that in its original form is due to Mazur [12]. It is a theorem for the category of all P.L. maps (not just proper ones); see [15, $\$ 0$ and $\$ 2]$ for more discussion.

Theorem 8 (The Tangential Equivalence Principle). Let $f: M_{1} \rightarrow M_{2}$ be $a$ (not necessarily proper) homotopy equivalence of unbounded P.L. n-manifolds such that $n \geqq 5$, and

(1) if $\tau\left(M_{1}\right), \tau\left(M_{2}\right)$ are the tangent P.L. microbundles, $f^{*} \tau\left(M_{2}\right)$ is stably isomorphic as a microbundle to $\tau\left(M_{1}\right)$;

(2) $M_{1}$ and $M_{2}$ have spines of dimension $<n / 2$ (i.e., $M_{1}$ and $M_{2}$ are the interiors of $n$-thickenings of complexes of dimension $<n / 2)$; then $f$ is homotopic to a P.L. homeomorphism (the homotopy being perhaps not proper).

REMARK. The examples given after the proof of the theorem show that the dimension condition on the spines cannot be improved.

Proof of Theorem. Let $X_{k} \hookrightarrow T_{k}, k=1$ or 2 , be $n$-thickenings of polyhedra $X_{k}$ of dimension $<n / 2$ such that $M_{k}=\operatorname{int} T_{k} \supset X_{k}$. By general position techniques it can be assumed that $f\left(X_{1}\right) \cap X_{2}=\varnothing$. Let $N$ be a regular neighbourhood of $X_{2}$ in $M_{2}$ so small that $N \cap f\left(X_{1}\right)=\varnothing$. Then $M_{2}-$ int $N$ can be identified with $\partial N \times[0, \infty)$. For any proper P.L. map $\alpha: M_{1} \rightarrow[0, \infty)$ there is an obvious (not necessarily proper) homotopy of $f \mid X_{1}$ to a map $f^{\prime}: X_{1} \rightarrow M_{2}-$ int $N \cong \partial N \times[0, \infty)$ such that

$\left.{ }^{4}\right)$ Solutions have been claimed but apparently without justification (cf. [20]). For further discussion see [14]. 
$f^{\prime}$ has component $\alpha$ on $[0, \infty)$. This $f^{\prime}$ is proper since $\alpha$ is proper. Moving $f^{\prime}$ slightly to general position arranges that $f^{\prime}$ is a proper P.L. imbedding. By the open regular neighbourhood identification theorem [15, \$2]-which applies because $\operatorname{dim} M_{2} \geqq 5$ and $\operatorname{dim} X_{1}+3 \leqq \operatorname{dim} M_{2}-M_{2}$ can be identified with int $T_{1}^{\prime}$ for some thickening

$$
X_{1} \stackrel{f^{\prime}}{\longrightarrow} M_{2} \longleftrightarrow T_{1}^{\prime} .
$$

The tangentiality condition (1), and Theorem 7 , Corollary 2, imply that this thickening is equivalent to $X_{1} \hookrightarrow T_{1}$. Hence there is a P.L. homeomorphism $h$ : int $T_{1} \rightarrow \operatorname{int} T_{1}^{\prime}$ with $h \mid X_{1}=f^{\prime}$, so that $h$ is homotopic to $f$.

The remainder of this section is devoted to some examples. An elementary lemma will be required:

LEMMA 4. Let $M^{2 k}$ be an unbounded oriented topological manifold of dimension $2 k$. If $M^{2 k}=X \times R$ for any topological space $X$, then all intersection numbers $x \cdot y$ are zero for $x, y \in H_{k}\left(M^{2 k} ; Z\right)$.

Proof. By definition, $x \cdot y$ is the Kronecker product $\langle D x, y\rangle$ where $D x$ $\in H_{c}^{k}\left(M^{2 k} ; Z\right)$ is the Poincare dual of $x$, and $H_{c}$ denotes cohomology with compact supports. Thus, if $c^{k}$ is a singular cochain with compact support $K$ representing $D x$ and $d_{k}$ is a singular cycle representing $y, x \cdot y=c^{k}\left(d_{k}\right)$. Now, if $M=X \times R$, $d_{k}$ can be chosen to have support outside $K$, and hence $x \cdot y=c^{k}\left(d_{k}\right)=0$.

EXAMPLES. Let $M_{1}$ be a regular neighbourhood of the wedge $S^{k} \vee S^{k}$ in $S^{k} \times S^{k}$. Let $M_{2}$ be $D^{2 k} \cup$ \{two trivially attached $k$-handles\}. Then $M_{2}$ also has a copy of $S^{k} \vee S^{k}$ as a spine-namely the union of the handle cores with the cone, in $D^{2 k}$, on the two attaching spheres.

(a) By the usual geometric interpretation, the intersection number of the two spheres of the wedge in $M_{1}$ is \pm 1 . Hence int $M_{1} \neq X \times R$ for any $X$ by Lemma 4, and thus

$$
S_{2 k-1}: \mathscr{T}^{2 k-1}\left(S^{k} \vee S^{k}\right) \rightarrow \mathscr{T}^{2 k}\left(S^{k} \vee S^{k}\right)
$$

is not surjective.

(b) Stabilizing the thickenings $S^{k} \vee S^{k} \hookrightarrow M_{1}$ and $S^{k} \vee S^{k} \hookrightarrow M_{2}$ by crossing with $[-1,1]$ gives equivalent $(2 k+1)$-thickenings of $S^{k} \vee S^{k}$ (to see this use Theorem 7, Corollary 2 or argue directly). But the $2 k$-thickenings are distinct since $M_{2}=N \times I$, where $N$ is a $(2 k-1)$-manifold formed by adding two trivially attached $k$-handles to a $(2 k-1)$-ball, and, by (a), $M_{1} \neq X \times I$ for any $X$. Thus

$$
s_{2 k}: \mathscr{T}^{2 k}\left(S^{k} \vee S^{k}\right) \rightarrow \mathscr{T}^{2 k+1}\left(S^{k} \vee S^{k}\right)
$$

is not injective.

(c) There is, by (b), a homotopy equivalence $f$ : int $M_{1} \rightarrow \operatorname{int} M_{2}$ covered by a map of the (trivial) stable tangent bundles. Yet int $M_{1}$ and int $M_{2}$ are not homeomorphic, since only one of them is a product of the form $X \times R$. Hence the dimension condition on the spines in Theorem 8 cannot, in general, be improved. 
5. Relative versions. A theorem lacking a relative version is an impotent thing, for it is a common experience that only the relative form of a theorem proves useful. We are fortunate in that a relative version of our main tool, Theorem 1 , is a fairly easy consequence of the original absolute version. Relative versions of the other results of this paper can then be produced by mimicking the original proofs. For the relative Theorem 1 we give full details, but for the other results we are content to assist with a few remarks.

Here is the neatest relative form of Theorem 1. Later we will deduce a more straightforward one that is slightly more general. ([1] is a general reference for results on relative regular neighbourhoods which are used extensively in this section.)

THEOREM 1(r). Let $X$ be a polyhedron and suppose $X \times I$ is imbedded in an $n$ manifold without boundary $M$, with $n \geqq \operatorname{dim}(X \times I)+3$. Let $Y$ be a closed subpolyhedron of $X$. Consider a relative regular neighbourhood $P$ in $M$ of $X \times I$ modulo $(X \times \partial I) \cup(Y \times I)$. Let $P_{0}$ be a relative regular neighbourhood in $\partial P$ of $X \times 0$ modulo $(Y \times I) \cup(X \times 1)$, and $P_{1}$ be a regular neighbourhood of $X \times 1$ in $\partial P$ modulo $(Y \times I)$ $\cup P_{0}$. Then there exists a homeomorphism $f: P_{0} \times I \rightarrow P$ such that for $(x, 0) \in X$ $\times 0 \subset P_{0}, f((x, 0), t)=(x, t) \in X \times I \subset P, f \mid P_{0} \times 0$ gives $P_{0} \hookrightarrow P$, and $f\left(P_{0} \times 1\right)=P_{1}$.

Proof. If $Y=\varnothing$, an application of Theorem 1 to $X \times I \subset P$ together with regular neighbourhood uniqueness provides the desired $f$. (Note that $P$ is a manifold when $Y=\varnothing$.)

To prove the general case let $P^{\prime}, P_{0}^{\prime}$ and $f^{\prime}: P_{0}^{\prime} \times I \rightarrow P^{\prime}$ be obtained by the special case of the theorem with $\varnothing$ in place of $Y$ but $X \times I \subset M$ as given. Observe that, by regular neighbourhood uniqueness, the theorem is true for any choice of $P$ and $P_{0}$ if it is true for one choice of $P$ and $P_{0}$. Hence if $Q_{0}$ denotes a regular neighbourhood in $P_{0}^{\prime}$ of $X \times 0$ modulo $Y \times 0$, it suffices to show that $f^{\prime}\left(Q_{0} \times I\right)$ is a regular neighbourhood in $M$ of $X \times I$ modulo $(X \times \partial I) \cup(Y \times I)$. For then $\left(f^{\prime}\left(Q_{0} \times I\right), Q_{0}\right)$ is a choice of $\left(P, P_{0}\right)$ so that the theorem holds with $f=f^{\prime} \mid Q_{0} \times I$. That $f^{\prime}\left(Q_{0} \times I\right)$ is indeed such a neighbourhood is an immediate consequence of Cohen's identification theorem [1, Theorem 6.1(b)]. Thus Theorem 1(r) is proved.

The following corollary of Theorem $1(\mathrm{r})$ is a many-parameter version of Theorem 1.

THEOREM 9. Let $X \times D^{s}$ be a subpolyhedron of an n-manifold without boundary $M^{n}$ such that $n \geqq \operatorname{dim}\left(X \times D^{s}\right)+3$. Let $P$ be a regular neighbourhood of $X \times D^{s}$ modulo $X \times \partial D^{s}$. Then there exists an $(n-s)$-thickening $Q$ of $X$ and a homeomorphism $f: Q \times D^{s} \rightarrow P$ such that for $x \in X \subset Q, f(x, t)=(x, t) \in X \times D^{s} \subset P$.

Proof (by induction on $s$ ). Write $D^{s}=D^{s-1} \times I$ and $X^{\prime}=X \times D^{s-1}$. Applying Theorem 1(r) to $X^{\prime} \times I \subset M$ with the subset $X \times \partial D^{s-1}$ of $X^{\prime}$ distinguished, we find a product structure $P=P_{0} \times I$ on $P$ agreeing with that on $X^{\prime} \times I$ so that $P_{0} \times 0$ is a 
regular neighbourhood in $\partial P$ of $X^{\prime} \times 0$ modulo $\left(\left(X \times \partial D^{s-1}\right) \times I\right) \cup X^{\prime} \times 1$ and a fortiori modulo $\left(X \times \partial D^{s-1}\right) \times 0$. Now $P$ is an $n$-manifold, so $\partial P$ is an $(n-1)$ manifold. The theorem for $(s-1)$ provides a thickening $X \hookrightarrow Q$ and a product structure $P_{0}=Q \times D^{s-1}$ agreeing with that on $X^{\prime}=X \times D^{s-1}$. Hence $P=Q \times D^{s}$, the product structure agreeing with that on $X \times D^{s} \subset P$, as was required.

Next we deduce the most simpleminded relativization of Theorem 1 . Let $C=\left(W ; V_{0}, V_{1}\right)$ be a cobordism of P.L. manifolds with boundary. This means that if $\delta W=$ closure $\left(\partial W-\left(V_{0} \cup V_{1}\right)\right)$, then $\delta W$ gives a cobordism (without boundary) $\delta C=\left(\delta W ; \partial V_{0}, \partial V_{1}\right)$ from $\partial V_{0}$ to $\partial V_{1}$. Let $(X, Y)$ be a polyhedral pair and suppose that $X \times I$ is imbedded in $W$ in such a way that $(X \times I) \cap \delta W=Y \times I$, and $(X \times I) \cap V_{t}=X \times t$ for $t=0,1$. Let $P$ be a regular neighbourhood in $W$ of $X \times I$ such that if $\delta P=P \cap \delta W$ and $P_{t}=P \cap V_{t}$, then $P_{t}$ is a regular neighbourhood of $X \times t$ in $V_{t}$ meeting $\partial V_{t}$ regularly (see [8]) for $t=0$, 1, and that $\delta P$ is a regular neighbourhood of $Y \times I$ in $\delta W$ meeting $\partial \delta W$ regularly. Note that a second derived neighbourhood in some triangulation of the triple $\left(W, V_{0}, V_{1}\right)$ provides such a $P$. The following theorem refers to the above notation.

THEOREM $1\left(\mathrm{r}^{\prime}\right)$. If $\operatorname{dim} W=n \geqq \operatorname{dim}(X \times I)+3$ and $(n-1) \geqq \operatorname{dim}(Y \times I)+3$, then there exists a homeomorphism $f: P_{0} \times I \rightarrow P$ such that:

(i) $\operatorname{For}(x, t) \in X \times I, f((x, 0) \times t)=(x, t)$.

(ii) $f\left(\left(P_{0} \cap \partial V_{0}\right) \times I\right)=\delta P$ and $f\left(P_{0} \times t\right)=P_{t}$ for $t=0,1$.

Proof. Let $W^{+}=W \cup(\delta P \times[0,1])$ with $p \in \delta P$ identified with $(p, 0) \in \delta P \times[0,1]$. $W^{+}$is a manifold containing $X^{+} \times I$ where $X^{+}=X \cup(Y \times[0,1]), y \in Y$ being identified with $(y, 0) \in Y \times[0,1]$. Let $P^{+}=P \cup(\delta P \times[0,1])$, let $V_{t}^{+}=V_{t} \cup$ $\left(\left(\delta P \cap \partial V_{t}\right) \times[0,1]\right)$ for $t=0,1$, and $P_{t}^{+}=P^{+} \cap V_{t}^{+}$. Then $P_{t}^{+}$is a regular neighbourhood of $X^{+} \times t$ modulo $(Y \times 1) \times I$ in $\partial P^{+}$. Now let $W^{++}$be $W^{+}$enlarged by adding a collar along $\partial W^{+}$, then $P^{+}$is a regular neighbourhood, in $W^{++}$, of $X^{+} \times I$ modulo $\left(X^{+} \times \partial I\right) \cup((Y \times 1) \times I)$. (Note. this would not have been true if the "plus" notation had not been introduced.) By Theorem 1(r) there is a homeomorphism $f^{+}: P_{0}^{+} \times I \rightarrow P^{+}$such that $f^{+}((x, 0) ; t)=(x, t)$ for all $(x, t) \in X^{+} \times I$, $f^{+}\left(P_{t}^{+} \times 0\right)=P_{t}$ for $t=0,1$. Now for $t=0,1$, the closure of $\left(V_{t}^{+}-V_{t}\right)$ is a regular neighbourhood $R_{t}$ of $(Y \times[0,1]) \times t$ in $P_{t}^{+}$modulo $X \times t$, meeting $\partial P_{t}^{+}$regularly. $f^{+}\left(R_{0} \times t\right)$ is another such neighbourhood and so by regular neighbourhood uniqueness, for either $t, f^{+}\left(R_{t}\right)$ is ambient isotopic to $R_{t}$ in $P_{t}^{+}$keeping $X^{+} \times t$ fixed. Using the product structure given by $f^{+}$on $P^{+}$, the isotopies can be extended into $P^{+}$, so that it can be assumed that $f^{+}$originally had the property that $f^{+}\left(R_{0} \times t\right)=R_{t}$ for $t=0$ and 1 . Now we repeat the same type of trick. $\delta P \times[0,1]$ is a regular neighbourhood in $P^{+}$of $((Y \times[0,1]) \times I) \cup R_{0} \cup R_{1}$ modulo $(X \times I)$ $\cup P_{0} \cup P_{1}$ meeting $\partial P^{+}$regularly; $f^{+}\left(R_{0} \times I\right)$ is another such neighbourhood. Thus using uniqueness again, it may be assumed that, after an isotopy (kceping $\left(X^{+} \times I\right) \cup P_{0}^{+} \cup P_{1}^{+}$fixed $), f^{+}\left(R_{0} \times I\right)=\delta P \times[0,1]$. Then let $f=f^{+} \mid P_{0} \times I$, $f\left(P_{0} \times I\right)=P$, and properties (i) and (ii) are easily verified. 
We now come to the question of giving relative versions of the results in $\$ \S 2-5$. The recipe is simple: the relative version of each result is the best generalization obtained by following the original line of argument and using Theorem 1( $\left.\mathrm{r}^{\prime}\right)$ in place of Theorem 1. Occasionally one finds that, as with Theorem 1, the relative version can be directly deduced from the original version. We will say little more about proofs but give some help section by section with statements:

On $\S 2$. Here are the changes in Theorem 2 on pseudo-isotopy extension (the changes in Theorem 3 are analogous). Imbeddings of a polyhedral pair $(X, Y)$ (with $Y$ closed in $X)$ into $(Q, \partial Q)$ are treated. The pseudo-isotopy is a given imbedding $e: X \times I \rightarrow Q \times I$ such that $e^{-1}(\partial Q \times I)=Y \times I$ and $e^{-1}(Q \times \partial I)=X \times \partial I$. To the assumptions add $\operatorname{dim} Y+3 \leqq \operatorname{dim} \partial Q$. The rest of the statement is unchanged.

On §3. The existence and uniqueness theorems for regular neighbourhoods associated with an immersion admit drastic relativization having nothing to do with Theorem 1. Roughly stated, if the target space is equipped with a finite family of closed subpolyhedra, then the regular neighbourhood associated with the immersion comes equipped with a corresponding family of subpolyhedra. In uniqueness and invariance statements the subpolyhedra are respected wherever conceivable. We leave this notion aside since any case we use is trivial (but see Cohen [1]).

A relative version of the Corollary to Theorem 6 can be proved from Theorem $1\left(\mathrm{r}^{\prime}\right)$. This shows that immersions of $(X, Y)$ in $(Q, \partial Q)$ which are (pairwise) pseudo-regularly homotopic have equivalent associated regular neighbourhoods if $\operatorname{codim}(Q, X)$ and $\operatorname{codim}(\partial Q, Y)$ are $\geqq 3$.

Definitions. An $n$-thickening of a polyhedral pair $(X, Y)$ is an imbedding $e:(X, Y) \rightarrow(T, U)$ so that:

(1) $T$ is an $n$-manifold and $U$ is a (n-1)-submanifold (with boundary) of $\partial T$.

(2) $e^{-1}$ (int $\left.U\right)=Y$ and $e X \subset$ int $T \cup$ int $U$.

(3) $(T, U)$ is P.L. homeomorphic fixing $e X$ to $(P, P \cap \partial T)$ for a regular neighbourhood $P$ of $e X$ in $T$.

Another $n$-thickening $e^{\prime}:(X, Y) \rightarrow\left(T^{\prime}, U^{\prime}\right)$ of $(X, Y)$ is called equivalent to $e$ if there exists a pair isomorphism $\phi:(T, U) \rightarrow\left(T^{\prime}, U^{\prime}\right)$ so that $\phi e=e^{\prime}$.

The set of equivalence classes of $n$-thickenings of $(X, Y)$ is written $\mathscr{T}^{n}(X, Y)$. The assignment to an $n$-thickening $e:(X, Y) \rightarrow(T, U)$ of $e \times 0:(X, Y) \rightarrow(T, U)$ $\times[-1,1]$ gives the stabilization map $s_{n}: \mathscr{T}^{n}(X, Y) \rightarrow \mathscr{T}^{n+1}(X, Y)$. Then if $\operatorname{dim}(X, Y)$ is defined to be $\max (\operatorname{dim} X, \operatorname{dim} Y+1 / 2)$ the relative stability theorem is: $s_{n}$ is onto for $n \geqq 2 \operatorname{dim}(X, Y)$ and injective for $n>2 \operatorname{dim}(X, Y)$. For $n>$ $2 \operatorname{dim}(X, Y), \mathscr{T}^{n}(X, Y)=\left[X, B_{\mathrm{PL}}\right]-\operatorname{not}\left[X / Y, B_{\mathrm{PL}}\right]$. If $f:\left(M_{1}, \partial M_{1}\right) \rightarrow\left(M_{2}, \partial M_{2}\right)$ is a nonproper pairwise homotopy equivalence of $n$-manifolds with boundary that is covered by a map of stable tangent (micro-) bundles, then $f$ is homotopic to a homeomorphism of pairs provided $n \geqq 6$, and $\left(M_{i}, \partial M_{i}\right)$ is (int $T_{i}$, int $\left.U_{i}\right), i=1,2$, 
for some $n$-thickening $\left(X_{i}, Y_{i}\right) \hookrightarrow\left(T_{i}, U_{i}\right)$ with $\operatorname{dim}\left(X_{i}, Y_{i}\right)<n / 2$. The same holds for $n=5$ at least if we assume that $f \mid \partial M_{1}$ is a homeomorphism onto $\partial M_{2}$. The dimension restrictions in this last theorem come from Theorem 2.1 of [15].

6. Smooth versions. One could define smooth regular neighbourhoods for $C^{\mathbf{1}}$ subpolyhedra of smooth manifolds and prove existence and uniqueness theorems as in the P.L. case. See Hirsch [3]. Then one might translate all our P.L. results to smooth manifolds. But there is a complication. The translated results would hold only up to a suitable concordance. For example, consider an interval imbedded in the shape of a $W$ in the 4-disc with its end points on the boundary. A literal translation of Theorem 1 would say that the interval is smoothly imbedded, whereas it has in fact three corners. This complication makes translation of $\$ \$ 1-3$ seem fruitless. However the stability results of $\S 4$ have pleasant translations. We give them even in relative form.

A smooth n-thickening of a polyhedral pair $(X, Y)$ is a P.L. $n$-thickening $e:(X, Y) \hookrightarrow(T, U)$ equipped with a smoothness structure $\alpha$ on $T^{\circ}=$ int $T \cup$ int $U$ that is compatible with the P.L. structure. One smooth $n$-thickening $(e, \alpha)$ of $(X, Y)$ is said to be smoothly equivalent to another $\left(e^{\prime}, \alpha^{\prime}\right)$ (distinguished by primes) if there is a P.L. homeomorphism $h:(T, U) \rightarrow\left(T^{\prime}, U^{\prime}\right)$ so that $e^{\prime}=h e$ and the smoothing $h^{*} \alpha^{\prime}$ of $T^{\circ}$ is concordant to $\alpha$. The set of smooth equivalence classes of smooth $n$-thickenings of $(X, Y)$ is denoted by $\mathscr{S T}^{n}(X, Y)$ and, as usual, the stabilizing map

$$
s_{n}: \mathscr{S T}^{n}(X, Y) \rightarrow \mathscr{S T}^{n+1}(X, Y)
$$

comes from taking products with $[-1,1]$.

REMARK 1. One might consider definitions of smooth thickening and smooth equivalence which differ from the above in that $\alpha$ is a smoothing of all of $T$, or perhaps of int $T$. In fact all the three definitions of $\mathscr{S T}^{n}(X, Y)$ are equivalent under the "forgetting" map on smoothings from $T$ to $T^{\circ}$ to int $T$. This follows from the Cairns-Hirsch theorem [4]. Smoothing $T^{\circ}$ combines two technical advantages: product with $[-1,1]$ introduces no corners, and there is an induced smooth thickening $(e|Y, \alpha|$ int $U)$.

REMARK 2. It is known (cf. [9]) that the smoothings of a P.L. manifold $M$ with a given base smoothing $\alpha_{0}$ are classified functorially up to concordance by homotopy classes of maps of $M$ to an $H$-space called PL/O. Thus if $f: N \rightarrow M$ is a map of P.L. manifolds and $\alpha$ is a smoothing of $M$, there is an induced smoothing $f^{*} \alpha$ of $N$ determined to concordance. The rule is natural in the sense that:

(a) If $f$ is an open P.L. imbedding, $f^{*} \alpha$ is the restricted smoothing.

(b) If $M=N \times R^{s}$, and $f(n)=(n, 0), f^{*} \alpha$ is the smoothing $\beta$ on $N$ unique to concordance such that $\beta \times R^{s}$ is concordant to $\alpha$ (see [4, Theorem 2.1]).

Thus $\alpha$ and $\alpha^{\prime}$ in the above definition determine elements $[\alpha],\left[\alpha^{\prime}\right]$ in $[X$, PL/O] and $h^{*} \alpha^{\prime}$ is concordant to $\alpha$ if and only if $[\alpha]=\left[\alpha^{\prime}\right]$. 
TheOREM 9. If $\operatorname{dim}(X, Y)$ denotes $\max (\operatorname{dim} X, \operatorname{dim} Y+1 / 2)$, then

$$
s_{n}: \mathscr{S T}^{n}(X, Y) \rightarrow \mathscr{S}^{n+1}(X, Y)
$$

is surjective for $n \geqq 2 \operatorname{dim}(X, Y)$ and injective for $n>2 \operatorname{dim}(X, Y)$.

Proof. Surjectivity follows from the P.L. version and the Cairns-Hirsch theorem [4, Theorem 2.1]. Injectivity follows immediately from the P.L. case and Remark 2-because the classifying map for a smoothing $\alpha \times(-1,1)$ agrees with that for $\alpha$. (Alternately, one can use the Cairns-Hirsch theorem again.)

Corollary. For $n>2 \operatorname{dim}(X, Y), \mathscr{S}^{n}(X, Y) \cong\left[X, B_{O}\right]$.

Proof (Noticeably harder than the P.L. proof). Let there be given P.L. $\boldsymbol{n}$-thickenings $e:(X, Y) \hookrightarrow(T, U), e^{\prime}:(X, Y) \hookrightarrow\left(T^{\prime}, U^{\prime}\right)$ of $(X, Y)$ equipped with compatible smoothings $\alpha, \alpha^{\prime}$ on all of $T, T^{\prime}$ respectively (cf. Remark 1). The smoothings $\alpha, \alpha^{\prime}$ are easily made to have corners along $\partial U, \partial U^{\prime}$ with model $Q=\left\{\boldsymbol{x} \in \boldsymbol{R}^{n}: x_{1} \geqq 0\right.$, $\left.x_{2} \geqq 0\right\}$. Thus, for example, each point of $T$ has a neighbourhood diffeomorphic to an open subset of the model $Q$, and $\partial U$ is exactly the set of points of $T$ that must correspond to points $x \in Q$ with $x_{1}=x_{2}=0$. Suppose that the tangent bundle classifying maps to $B_{O}$ of $\alpha, \alpha^{\prime}$ are homotopic. Then in view of the above theorem our task is to show that the smooth thickenings become smoothly equivalent after sufficient stabilizing. This will establish the corollary, since the classification map $\mathscr{S} \mathscr{T}(X, Y) \rightarrow\left[X, B_{O}\right]$ is onto by the argument used for the P.L. version in $\S 4$.

Take a proper retraction $r:(T, U) \rightarrow(X, Y), r e=1$, and let $f$ be a $\operatorname{good}\left(^{5}\right)$ smooth imbedding approximating $\left(e^{\prime} \times 0\right) r:(T, U) \rightarrow\left(T^{\prime}, U^{\prime}\right) \times D^{s}, s \geqq n+1$, and properly homotopic to it. Then the normal bundle of $f$ is trivial because $\alpha, \alpha^{\prime}$ have the same stable tangent bundle over $X$. It follows that $f$ extends to a smooth imbedding $F:(T, U) \times D^{s} \rightarrow\left(T^{\prime}, U^{\prime}\right) \times D^{s}$ with $F(x, 0)=f(x)$ for $x \in T$, onto a closed neighbourhood of $e(X) \times 0$ small enough so that it does not intersect $\partial\left(T^{\prime} \times D^{s}\right)$-int $\left(U^{\prime} \times D^{s}\right)$. Changing notation we can assume that $s=1$ and $\operatorname{dim}(X, Y)$ is much less than $n / 2$. For a future application of Theorem $1\left(\mathrm{r}^{\prime}\right)$, let $(W, \delta W)=\left(T^{\prime}, U^{\prime}\right) \times D^{1}-F\left\{\left(T^{\circ}\right.\right.$, int $\left.U\right) \times$ int $\left.D^{1}\right\}$ and let $V=W \cap F\left(T \times D^{1}\right)$, $V^{\prime}=\partial\left(T^{\prime} \times D^{1}\right)$-int $\left(U^{\prime} \times D^{1}\right)$. Then $\left(W ; V, V^{\prime}\right)$ is a cobordism with boundary because $F\left(T \times D^{s}\right) \cap\left[\partial\left(T^{\prime} \times D^{s}\right)\right.$-int $\left.\left(U^{\prime} \times D^{s}\right)\right]=\varnothing$. Extend a triangulation of $V \cup V^{\prime}$ inherited from P.L. triangulations of $T, T^{\prime}$, to a triangulation of $W$ that is $C^{1}$ for the smoothing $\alpha^{\prime} \times D^{1}$ of $T^{\prime} \times D^{1}$.

By a general position argument, the map $F(e \times 1)$ of $(X, Y)$ to $(V, \partial V)$ is homotopic in $(W, \delta W)$ to the map $e^{\prime} \times 1$ into $\left(V^{\prime}, \partial V^{\prime}\right)$ and we can assume that the homotopy $\phi$ is an imbedding of $X \times I$ P.L. with respect to the above $C^{1}$ triangulation, that has the features needed to apply Theorem $1\left(\mathrm{r}^{\prime}\right)$. Then apply Theorem $1\left(\mathrm{r}^{\prime}\right)$ to a regular neighbourhood $P$ of the imbedded $X \times I$ such that $P \cap V=F(T \times 1)$ and $P \cap V^{\prime}$

$\left({ }^{5}\right)$ A good imbedding of pairs $f:(A, B) \rightarrow(C, D)$ is an imbedding such that $f^{-1} D=B$. 
$=T^{\prime} \times 1$. Thus we find a P.L. homeomorphism $h: T \rightarrow T^{\prime}$ with $h e=e^{\prime}$, and a concordance from $\alpha$ to $h^{*} \alpha^{\prime}$-both arising from the P.L. product structure $P \cong(P \cap V) \times I$ provided by Theorem $1\left(\mathrm{r}^{\prime}\right)$. This shows that the smooth thickening

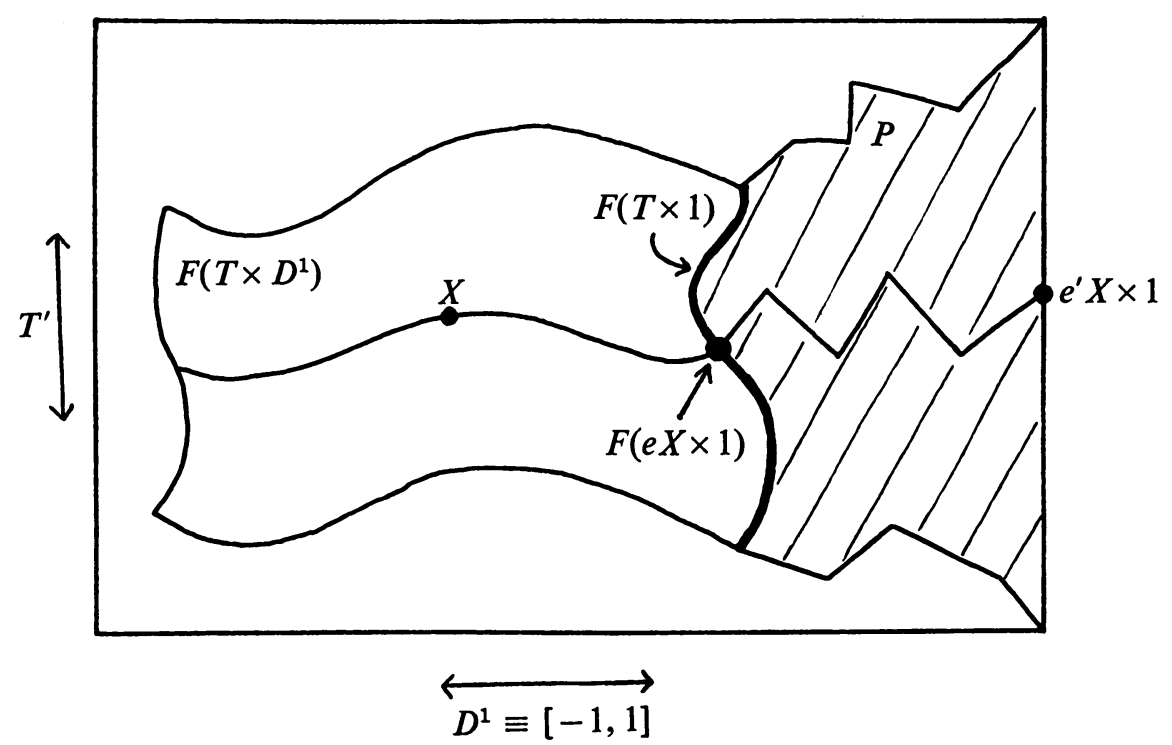

$(e, \alpha)$ is smoothly equivalent to $\left(e^{\prime}, \alpha^{\prime}\right)$. Since these are stabilizations of the smooth thickenings (of the same name) that we set out with, the corollary is established.

Finally we observe that the important tangential equivalence principle holds for the category of smooth manifolds and smooth maps in the relative form stated in the last paragraph of $\S 5$. In the statement, tangent vector bundle replaces tangent P.L. microbundle and diffeomorphism replaces P.L. homeomorphism. The proof imitates the brief argument given in $\S 4$ for the absolute P.L. version; the above corollary takes the place of the parallel corollary $\mathscr{T}^{n}(X) \cong\left[X, B_{\mathrm{PL}}\right]$ of $\S 4$, and the theorem 'concordance $\Rightarrow$ diffeomorphism' [4] of Munkres and Thom is used.

\section{Appendix}

The following proposition completes the proof of Theorem 7 (Stability of Thickenings) in the remaining exceptional case, namely, when $X$ is a 1-dimensional polyhedron.

Proposition 1. Let $X^{1}$ be a polyhedron of dimension 1 and $n$ be an integer $\geqq 3$. Then the operation of associating to each n-thickening of $X$ the first Steifel-Whitney class of its tangent bundle gives an isomorphism

$$
\mathscr{T}^{n}(X) \stackrel{w_{1}}{\longrightarrow} H^{1}\left(X ; Z_{2}\right)
$$

A lemma precedes the proof. 
LEMma. If $Y$ is a finite set of points and $X$ is the cone on $Y$, then $\mathscr{T}^{n}(X, Y)$ has just one element. It is represented by the cone on any imbedding of $Y$ in $S^{n-1}$.

Proof. Recall that, as defined in $\S 5, \mathscr{T}^{n}(X, Y)$ is the set of equivalence classes of $n$-thickenings of the pair $(X, Y)$. If $e:(X, Y) \rightarrow(T, U)$ is such a thickening, $T$ is, in this case, an $n$-ball (as $X$ is collapsible), and $U$ is a collection of disjoint (n-1)balls in $\partial T$, each containing one point of $e Y$. Further, $T$ is a regular neighbourhood of $e(X)$. If $n \geqq 4$, the result follows from the Cone Unknotting Theorem [10] together with regular neighbourhood uniqueness applied to $U$. For any $n$, the result can be deduced by noting that the pair $(T, e X)$ is homeomorphic to $(N, e X)$ keeping $e X$ fixed, where $N$ is a second derived neighbourhood of $e X$ in some triangulation of $T$. Then an exceedingly trivial application of the technique of proof in [10, Proposition 1] shows that $(N, e X)$ is homeomorphic to the cone on the pair (link $(e v, T)$, link $(e v, e X))$ where $v$ is the vertex of the cone $X$, and links are taken with respect to the above mentioned triangulation.

Proof of Proposition 1. Fix a triangulation of $X^{1}$ and choose in it a maximal tree $K(=$ maximal simply connected subcomplex $)$. Let $v_{1}, v_{2}, v_{3}, \ldots$ be vertices and let $S_{i}, L_{i}$ be the star and link of $v_{i}$ in the 2 nd barycentric subdivision. Let $\tilde{K}$ be the star of $K$ in this subdivision. Let $L=\bigcup_{i} L_{i}$ and $S=\bigcup_{i} S_{i}$. The closure of $X-S$ is a union of closed intervals. Let $I_{1}, I_{2}, I_{3}, \ldots$ be those in $\widetilde{K}$ and $J_{1}, J_{2}, J_{3}, \ldots$, be those not in $\tilde{K}$. Write $I=\bigcup_{i} I_{i}, J=\bigcup_{j} J_{j}$.

If $\partial \tilde{K}$ is the frantier of $\tilde{K}$ in $X$, let $(\tilde{K}, \partial \tilde{K}) \hookrightarrow(T, U)$ be any $n$-thickening of the pair $(\tilde{K}, \partial \tilde{K})$. Taking a regular neighbourhood $T_{0}$ in $T$ of $S$ modulo $I$, and then a regular neighbourhood $T_{1}$ of $I$ in $T$-int $T_{0}$ meeting $\partial\left(T\right.$-int $\left.T_{0}\right)$ regularly, we see that a copy of $T$ (namely $\left.T_{0} \cup T_{1}\right)$ is obtained by glueing an $n$-thickening of $(S, L)$ to an $n$-thickening of $(I, \partial I)$ along the boundary $(n-1)$-thickenings of $\partial I \subset L$. The lemma states that all the component thickenings are standard thickenings of pairs. Now any orientation preserving P.L. homeomorphism of a P.L. $n$-ball fixed on an interior point is isotopic to the identity keeping that point fixed (see [19]). Since $K$ is a tree, we can conclude that all n-thickenings of $(\tilde{K}, \partial \tilde{K})$ are equivalent.

Next let $X \hookrightarrow T$ be any $n$-thickening. Taking a regular neighbourhood $T_{0}$, in $T$, of $\tilde{K}$ modulo $J$, and then a regular neighbourhood $T_{1}$ of $J$ in $\left(T-\right.$ int $\left.T_{0}\right)$ meeting $\partial\left(T-\right.$ int $\left.T_{0}\right)$ regularly, we see that a copy of $T$ (namely $T_{0} \cup T_{1}$ ) is obtained by glueing an $n$-thickening $\left(T_{0}, U_{0}\right)$ of $(\tilde{K}, \partial \widetilde{K})$ to an $n$-thickening $\left(T_{1}, U_{1}\right)$ of $(J, \partial J)$ along the boundary $(n-1)$-thickening $U_{0}$ and $U_{1}$ of $\partial \widetilde{K}=\partial J$. By the lemma $\left(T_{1}, U_{1}\right)$ is a standard thickening, and by the preceding paragraph so is $\left(T_{0}, U_{0}\right)$. This time there are essentially different ways of pasting. In fact, if $B_{i}$ is the component of $T_{1}$ containing $J_{i}$, there are, by the isotopy property for homeomorphisms of P.L. balls quoted in the last paragraph, effectively exactly two ways of glueing $B_{i}$ to $T_{0}$ : one makes the loop determined by $J_{i}$ and the tree $\widetilde{K}$ orientation preserving, the other makes it orientation reversing. By "effectively" we mean up to P.L. homeomorphism of the identification spaces fixing points of $T_{0}$ and of $X$. It follows that 
the equivalence classes of thickenings of $X$ are in 1-1 correspondence with the functions that assign 0 or 1 to $J_{1}, J_{2}, J_{3}, \ldots$ The correspondence assigns to a thickening $X \hookrightarrow T$ the function which is 0 or 1 on $J_{i}$ according as the path in $T$ determined by $J_{i}$ and the tree is orientation preserving or reversing in $T$. But such functions form $\operatorname{Hom}\left(\pi_{1} X ; Z_{2}\right)=H^{1}\left(X ; Z_{2}\right)$ and the correspondence is $w_{1}$. This completes the proof.

\section{REFERENCES}

1. M. M. Cohen, A general theory of relative regular neighborhoods, Trans. Amer. Math. Soc. 136 (1969), 189-329.

2. A. Haefliger and V. Poenaru, La classification des immersions combinatoires, Inst. Hautes Études Sci. Publ. Math. 23 (1964), 75-91.

3. M. Hirsch, Smooth regular neighbourhoods, Ann. of Math. 76 (1962), 524-530.

4. - Obstruction theories for smoothing manifolds and maps, Bull. Amer. Math. Soc. 69 (1963), 352-356.

5. J. F. P. Hudson, Sunny collapsing, (to appear).

6. J. F. P. Hudson and W. B. R. Lickorish, Extending piecewise linear concordances (to appear).

7. J. F. P. Hudson and E. C. Zeeman, On combinatorial isotopy, Inst. Hautes Études Sci. Publ. Math. 19 (1964), 69-94.

8. — On regular neighbourhoods, Proc. London Math. Soc. (3) 14 (1964), 719-745.

9. R. Lashof and M. Rothenberg, Microbundles and smoothing, Topology 3 (1965), 357-388.

10. W. B. R. Lickorish, The piecewise linear unknotting of cones, Topology 4 (1965), 67-91.

11. J. Milnor, Lectures on the h-cobordism theorem, Princeton Math. Notes, Princeton Univ. Press, Princeton, N. J., 1965.

12. B. Mazur, Stable equivalence of differentiable manifolds, Bull. Amer. Math. Soc. 67 (1961), 377-384.

13. C. P. Rourke and B. J. Sanderson, Block bundles. I, Ann. of Math. (2) 87 (1968), 1-28.

14. J. J. Andrews and M. L. Curtis, Free groups and handlebodies, Proc. Amer. Math. Soc. 16 (1965), 192-195.

15. L. C. Siebenmann, On detecting open collars, Trans. Amer. Math. Soc. (to appear).

16. A. Scott, Infinite regular neighbourhoods, J. London Math. Soc. 42 (1967), 245-253.

17. C. T. C. Wall, Classification problems in differential topology. IV, Topology 5 (1966), 73-94.

18. - Piecewise linear normal micro-bundles, Bull. Amer. Math. Soc. 71 (1965), 638-641.

19. E. C. Zeeman, Seminar on combinatorial topology, Inst. Hautes Études Sci. notes, 19631965 .

20. —_, On the dunce hat, Topology 2 (1964), 341-358.

UNIVERSITY OF WISCONSIN,

MADISON, WISCONSIN

UNIVERSITÉ de PARIS,

91-OrSay, France 